\title{
Analysis of Atmospheric Effects on the Space-to-Ground Laser Communication Channel
}

\author{
Won-Ho Kim
}

\begin{abstract}
Space laser communication system has advantages such as very high bandwidth, inherent security, robustness to electromagnetic interference, unlicensed frequency band. However, space laser communication is affected by various factors. Atmospheric phenomena are major degradation factors of communication quality. The atmospheric loss is due to absorption, scattering and turbulence. In this paper, the atmospheric phenomena associated with degradation of communication quality are discussed and summarized to apply in channel modeling of space-to-ground laser communication systems.
\end{abstract}

Keywords - Space communication, Satellite communication, Laser communication, Free-space optical communication.

\section{INTRODUCTION}

Conventional radio communication has many limitations such as radio interference, transmission capacity, and communication security, while laser communication has many advantages such as robustness to electromagnetic interference, ultra-wideband, inherent security, and size-reduction. Current radio communication reaches the limit of massive data transmission capacity of satellite which increases rapidly every year, it is expected that the resolution of observing satellite sensors will increase gradually and the observation width and observation frequency will increase.

Recently, space or satellite laser communication has been put into practical use as a technical alternative to satellite radio communication [1]-[4]. Laser communication is the ultra-high speed communication system for transmitting and receiving data using laser. Attempts to apply lasers to space/satellite communications have not been long. Because of the advantage of high-speed communication from ground to space/satellite, studies are being actively conducted in advanced countries such as the United States (NASA), Europe (ESA) and Japan (NICT). Currently, NASA has completed $622 \mathrm{Mbps}$ data transmission experiment between lunar probe and ground using laser communication, and plans to carry out $2.88 \mathrm{Gbps}$ data transmission experiment in the future. This laser-based space/satellite communication technology can be used in various fields that require broadband data transmission such as weather observation, disaster monitoring, and environmental monitoring.

The major degradation of communication quality of space

Won-Ho Kim

Division of Electrical, Electronic and Control Engineering, Kongju National University, Cheonan, Republic of Korea. laser communication is the atmospheric phenomena such as absorption, scattering, and turbulence. In this paper, the atmospheric phenomena are discussed in detail to apply space laser communication channel modeling.

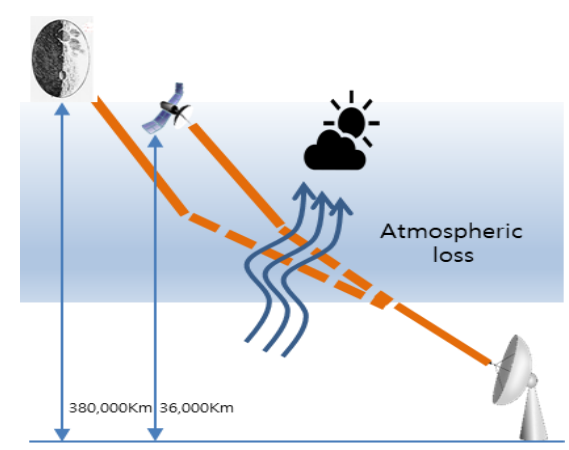

Fig. 1 Conceptual configuration of space/satellite-to-ground laser communication

\section{II.CHARACTERISTICS OF SPACE-TO-GROUND LASER COMMUNICATION}

Figure 1 shows conceptual configuration of space-to-ground laser communication. Space laser communication uses infrared rays in the range of $3 \mathrm{THz}$ to $3000 \mathrm{THz}$ for transmitting various kinds of information in a laser beam. The laser has strong directivity and straightness, and has weak barrier transmittance. The high frequency provides a wide bandwidth and enables high-speed transmission of observation data. The light has a single wavelength and is excellent in straightness, and high power, narrow beam width is maintained even in long distance transmission, and power efficiency is excellent as shown in figure 2 .

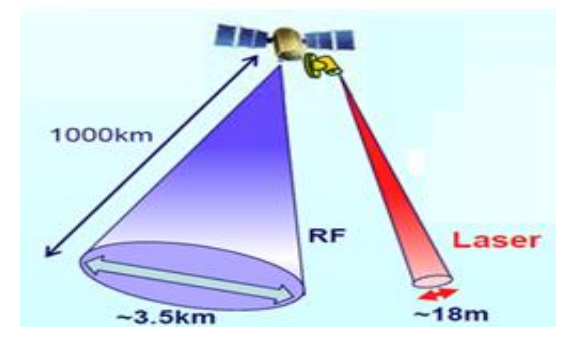

Fig. 2 Comparison of radio frequency and laser beam width

The frequency band of the laser is freely chosen because it is not related to the current ITU-R radio regulations and it can be 
chosen freely and is not subject to frequency adjustment between countries. If the beam is not pointed correctly, it is difficult to receive, so it has excellent communication security and also has excellent security against radio jamming, interference, and detection. Laser communication equipment can be manufactured with small size, light weight and low power consumption, and the performance can be improved by about $50 \%$ in weight and about $65 \%$ in power compared with RF device. Due to the sharp directivity of the laser beam, there is a disadvantage that precise acquisition, tracking and beam-directing are required between the communication systems. Signal fluctuations due to atmospheric phenomena (absorption, scattering, turbulence) occur and the performance of laser communication is degraded. Therefore, a countermeasure is necessary. Disadvantage factors of space laser communication are discussed in the following section.

\section{EFFECTS OF ATMOSPHERIC PHENOMENA ON LASER CHANNEL}

Transmission quality degradation factors of space/satellite-to-ground laser communication are various. These include system loss, geometric loss, misalignment loss, atmospheric loss. Major factors of quality loss are atmospheric absorption, atmospheric scattering and atmospheric turbulence. The absorption and scattering are the main atmospheric phenomena on the cloudy days, and absorption and turbulence are the main atmospheric phenomena on the clear days.

\section{A. Atmospheric Absorption}

When the optical signal passes through the atmosphere, energy absorption occurs by the particles such as $\mathrm{H}_{2} \mathrm{O}, \mathrm{CO}_{2}, \mathrm{O}_{2}$, $\mathrm{O}_{3}$, etc [5]. They absorb to some degree the laser light energy, causing and attenuation of the laser power. The absorption phenomenon of the light wave by the atmospheric particles can increase the transmission degree of the atmosphere by appropriately selecting the wavelength of the light source. Figure 3 shows the transmittance of the atmosphere according to the wavelength, and it can alleviate the atmospheric absorption by appropriately selecting the wavelength of the light source. Especially, light of near-IR band, absorption occurs primarily due to water particles.

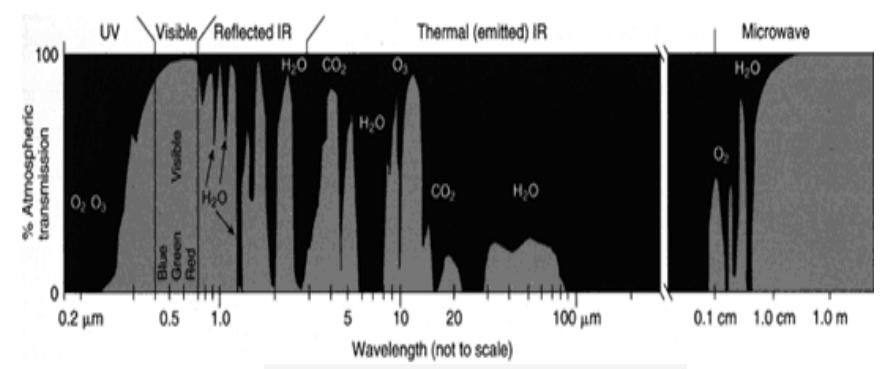

Fig. 3 Diagram of atmospheric windows [10]

Figure 3 shows wavelengths at which electromagnetic radiation penetrate the earth's atmosphere[10]. Chemical notation $\left(\mathrm{CO}_{2}, \mathrm{O}_{2}, \mathrm{O}_{3}\right)$ indicates the gas responsible for blocking sunlight at a particular wavelength. In the conventional satellite laser communication systems, $0.8,1.06,1.55 \mu \mathrm{m}$ bands have selected. Currently, to get high-bandwidth and interoperability with terrestrial system, $1.55 \mu \mathrm{m}$ band selection is being increased. Hence, $1.55 \mu \mathrm{m}$ of wavelength is appropriate in the channel modeling of space laser communication system.

\section{B. Atmospheric Scattering}

The scattering phenomenon occurs by the atmospheric particles such as rain, snow, fog, haze, pollution, dust, aerosols, smoke, etc. They cause light scattering, which is the deflection of incident light from its initial direction, causing spatial, angular, and temporal spread. Scattering strength is very high in condition that particle diameter is similar to the wavelength. The worst environmental conditions are fog and haze. They are composed of small particles with radius close to near-IR wave lengths. Typically, haze particles have a size between $0.01 \mu \mathrm{m}$ and $1 \mu \mathrm{m}$, and fog particles have a size between $1 \mu \mathrm{m}$ and $20 \mu \mathrm{m}[5]$.

The light scattering is classified into Rayleigh scattering and Mie scattering. Rayleigh scattering occurs when the size of the scattering particle is smaller than the wavelength of light. Rayleigh scattering is inversely proportional to the fourth power of the light wavelength, and the amount of light scattered decreases as the wavelength of light increases. It is mainly caused by small dust, nitrogen and oxygen molecules. Mie scattering is a phenomenon that occurs when the particle size is similar to the wavelength of light. It is mainly caused by smoke, fog and water vapor, and it affects longer wavelengths than Rayleigh scattering.
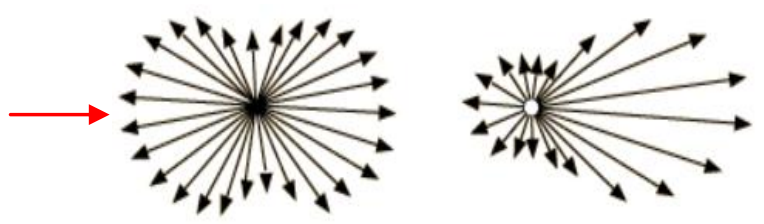

Fig. 4 Diagram of Rayleigh scattering(left) and Mie scattering(right)

As shown in figure 4, Rayleigh scattering occurs in various directions and Mie scattering proceeds in the direction of propagation. As the particle size becomes smaller, scattering occurs in the backward direction. When the size is about $0.05 \mu \mathrm{m}$, the intensity of light scattered in the direction opposite to the traveling direction becomes similar. In the case of Mie scattering, there is a characteristic of scattering in the direction of propagation, and the phenomenon becomes remarkable as the particle size increases. Mie scattering does not change scattering intensity depending on wavelength. All wavelengths are scattered almost evenly. The major scattering factor that affects the space laser communication channel is Mie scattering and it is applied in channel modeling of space laser communication system. 


\section{Atmospheric Turbulence}

Atmospheric turbulence is a phenomenon of variation in the refractive index of air caused by temperature changes, or variations in an arrival angle and wave-front distortion of light affected by wind, convection flow with wind or turbulent flow. The atmospheric turbulence causes random fluctuations in both the amplitude and the phase of the received signal. This is called channel fading. This is a considerable degradation of the communication performance, especially in long-distance transmissions such as space or satellite communication systems. The degradation of the communication quality is up to $20 \mathrm{~dB}$ [1].

Atmospheric turbulence is mainly characterized by three parameters. They are the inner and the outer scales of turbulence and the index of refraction structure parameter as called the turbulence strength. According to the Kolmogorov theory[5], outer scale is the largest cell size before the energy is injected into a region and inner scale is associated with the smallest cell size before energy is dissipated into heat. Configuration of received laser signal affected by of atmospheric turbulence is shown in figure 5[6]. The power spectrum of turbulence is calculated by convoluting a spatial frequency spectrum of speckle patterns in received beams caused by atmospheric turbulence and a spatial spectrum of a window function from aperture of an antenna. It is transformed into time-varying pseudo atmospheric turbulence signal and used in channel modeling of space laser communication system.

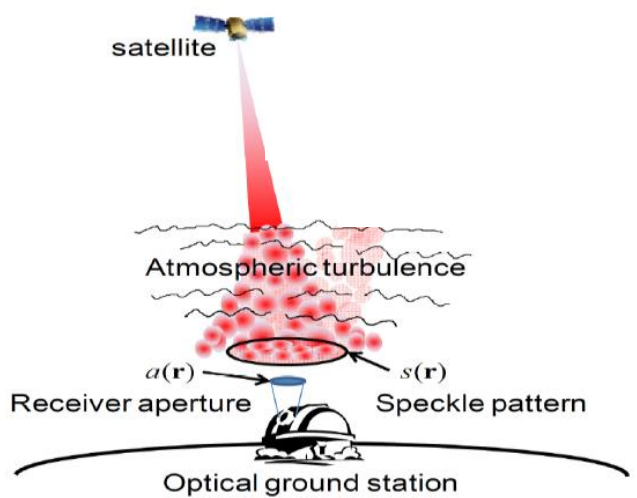

Fig. 5 Laser signal affected by atmospheric turbulence

\section{CONCLUSION}

In this paper, the atmospheric phenomena associated with degradation of communication quality are discussed and summarized to apply in channel modeling of space or satellite-to-ground laser communication. Major factors of quality loss are atmospheric absorption, atmospheric scattering and atmospheric turbulence in the laser transmission. The absorption loss can be avoided by appropriately selecting the wavelength of the light source. $1.55 \mu \mathrm{m}$ of wavelength is appropriate in the channel modeling. The major scattering factor that affects the space laser communication channel is Mie scattering. It is applied as major scattering factor in channel modeling. In addition, to mitigate scattering loss, site diversity method can be applied, and channel coding as like LDPC also can be solution. The atmospheric turbulence causes random fluctuations in both the amplitude and the phase of the received signal called channel fading. This is a considerable degradation of the communication performance. To alleviate quality degradation due to fading, powerful channel coding and modulation technique robust to fading can be applied. In the future, through channel modeling and simulation of laser communication, transmission scheme robust to atmospheric phenomena will be analyzed.

\section{ACKNOWLEDGMENT}

This research was supported by Basic Science Research Program through the National Research Foundation of Korea funded by the Ministry of Education (2017R1D1A1B03036302).

\section{REFERENCES}

[1] Sasaki Takashi, Toyoshima Morio, and Takenaka Hideki, "Fading Simulator for Satellite-to-Ground Optical Communication," Journal of the National Institute of Information and Communications Technology Vol. 59 Nos. 1/2, 2012.

[2] Okamoto Eiji, Shoji Yozo, Toyoshima Morio, and Takayama Yoshihi, "Transmission Experiments on OICETS Repeater Mode for Verification of Channel Coding Effect," ournal of the National Institute of Information and Communications Technology Vol. 59 Nos. 1/2, 2012.

[3] Nurizan binti Tahir, M. Naufal bin M. Saad, and Brahim Belhaouari Samir, "Binary Pulse Position Modulation (BPPM) Bit Error Rate (BER) Analysis in Turbulent Atmosphere," Journal of Telecommunication, Electronic and Computing Engineering, vol.2, No.1, 2010.

[4] Ales Vanderka, "Design, Simulation and Testing of the OOK NRZ Modulation Format for Free Space Optic Communication in a Simulation Box," Optics and optoelectronics, vol.12, No.6, 2014.

[5] Mohammad Ali Khalighi, "Survey on free space optical communication: A communication theory perspective," IEEE communication surveys \& Tutorials, Vol.16, No.4, 2014.

[6] Morio Toyoshima, Hideki Takenaka, Yoshihisa Takayama, "Atmosrheric turbulence-induced fading channel model for space-to-ground laser communications links," Optics express, Vol.19, No.17, 2011.

[7] Xiaoming Zhu and Joseph M. Kahn, "Free-Space Optical Communication through Atmospheric Turbulence Channels," IEEE Transactions on communications, VOL. 50, NO. 8, August 2002.

[8] Nikita J. Gaurihar, "Design and Implementation of LDPC codes and Turbo Codes using FPGA," International Research Journal of Engineering and Technology, Volume 03, Issue: 03, 2016.

[9] Tetsuya Kawanishi, "Ultra High-Speed LN Modulator Technology and its Applications," Opto-Electronics and Communications conference, 2011,

[10] https://earthobservatory.nasa.gov/Features/RemoteSensing/remote_04.p $\mathrm{hp}$

About Author (s):

Won-Ho Kim Ph.D/Professor, Division of Electrical, Electronic and Control Engineering, Kongju National University, Cheonan, Republic of Korea. His research interests are areas of multimedia satellite communication, image signal processing, communication signal processing. 